\title{
Morphological and functional alterations in patients with schizophrenia spectrum disorders
}

\author{
Andrea Schmitt $^{1} \cdot$ Peter Falkai $^{1}$
}

Published online: 9 December 2015

(C) Springer-Verlag Berlin Heidelberg 2015

In schizophrenia, the neurobiological difference between late and early onset of the disease remains unknown. In a 99mTc-ECD SPECT study, Wake et al. [1] found brain perfusion (rCBF) reduced in the precentral and inferior gyri in early-onset schizophrenia, while in late-onset patients, rCBF was reduced in the bilateral postcentral gyrus compared to healthy controls. Neurodevelopmental disturbances may underly morphological changes in the orbitofrontal cortex (OFC) in schizophrenia, but alterations in schizotypal disorder remain unknown. Nishikawa et al. [2] found a decrease in OFC sulcogyral pattern in schizophrenia patients compared to schizotypal patients and healthy controls, while olfactory sulcus depth in schizophrenia patients was decreased in schizophrenia < schizotypal patients $<$ healthy controls. The results suggest that schizotypal disorder might be a milder form of schizophrenia spectrum disorders, possibly reflecting common disease vulnerability. In a morphometric postmortem study, Bernstein et al. [3] found decreased volumes of the claustrum in patients with schizophrenia or major depression compared to healthy controls. Since this structure is highly connective, it is hypothesized that in schizophrenia, this deficit may contribute to impairments in sensory processing, while in major depression to disturbances in salience. In patients with schizophrenia, a dysfunction of the cerebellum has been related to neurological soft signs. Hirjak et al. [4] performed a structural and resting-state magnetic resonance imaging (MRI) investigation in 37 healthy young adults

Andrea Schmitt

Andrea.Schmitt@med.uni-muenchen.de

1 Department of Psychiatry and Psychotherapy, LudwigMaximilians-University Munich, Nußbaumstr. 7, 80336 Munich, Germany and analyzed the relationship between cerebellar structure and function and neurological soft signs. While they did not find a relationship between cerebellar volume and neurological soft signs, cerebellar lobule VI activity was associated with motor coordination and hard signs of the neurological soft sign domains. A negative relationship was found between lobule VI activity and the complex motor task domain. This cerebellar subregion has cortical sensorimotor projections. In a next step, this relationship should be assessed in schizophrenia patients.

In addition to neurological soft signs, alterations of exe movements have been described in schizophrenia. Dowiasch et al. [5] recorded eye movements in four different oculomotor tasks corresponding to natural everyday behavior in schizophrenia patients compared to healthy controls. Patients looking at predefined targets showed more fixations with reduced durations and had a greater root-mean-square error of retinal target velocity during visual tracking. The results obtained in a real environment differ from those derived in laboratory settings. This speaks for the ability of patients to compensate some deficits during natural behavior. In an electroencephalographic (EEG) study, Molina et al. [6] found that patients with schizophrenia displayed higher noise power and lower Shannon entropy compared to healthy controls. The results support the notion of an excess of gamma activity and a decreased modulation of EEG activity in schizophrenia.

Schizophrenia patients have a high rate of comorbid obsessive-compulsive disorder, whose symptoms have been associated with worse clinical outcome and greater disability. Tonna et al. [7] in their respective study found functioning to be positively related to the presence of mild obsessive-compulsive symptoms. However, they report an inverse relationship to moderate and severe symptoms. This relationship between functioning and obsessive-compulsive 
symptoms can be described by a reverse U-shaped curve. Neurocognitive assessments show that schizophrenia patients have deficits in Theory of Mind (ToM), which refers to the capacity to make inferences about one's own and others' thoughts and intentions. Zhang et al. [8] investigated 50 individuals with clinical high risk of psychosis with a neuropsychological test battery and social cognition tests. They re-assessed the probands after 12-month followup and examined the effects of baseline measurements on conversion to psychosis. Compared to healthy controls, the group with high risk of psychosis showed poorer neuropsychological performance. In converters to full-blown psychosis, these baseline scores were lower compared to non-converters. The authors suggest that declines in ToM ability and impairment in basic cognitive function may overlap with the progress of psychosis. In 67 individuals at risk of psychosis, $\mathrm{Xu}$ et al. [9] found that self-labelling as "mentally ill" predicted more positive attitudes toward psychiatric medication, In contrast, increased perceived stigma and the cognitive appraisal of stigma as a stressor predicted poorer attitudes toward psychotherapy. Early intervention could improve non-stigmatizing awareness of at-risk mental state to facilitate help-seeking. In search for biomarkers differentiating between schizophrenia and bipolar disorder, Hashimoto [10] discusses aberrant glutamate metabolism, $\mathrm{N}$-methyl-D-aspartate (NMDA) receptor hypofunction, and the effects of D-serine levels in brain regions in the pathogenesis of these disorders.

\section{References}

1. Wake R, Miyaoka T, Araki T, Kawakami K, Furuya M, Limoa E, Hashioka S, Horiguchi J (2015) Regional cerebral blood flow in late-onset schizophrenia: a SPECT study using 99mTc-ECD. Eur Arch Psychiatry Clin Neurosci. doi:10.1007/s00406-015-0607-z

2. Nishikawa Y, Takahashi T, Takayanagi Y, Furuichi A, Kido M, Nakamura M, Sasabayashi D, Noguchi K, Suzuki M (2015) Orbitofrontal sulcogyral pattern and olfactory sulcus depth in the schizophrenia spectrum. Eur Arch Psychiatry Clin Neurosci. doi:10.1007/s00406-015-0587-z

3. Bernstein HG, Ortmann A, Dobrowolny H, Steiner J, Brisch R, Gos T, Bogerts B (2015) Bilaterally reduced claustral volumes in schizophrenia and major depressive disorder: a morphometric postmortem study. Eur Arch Psychiatry Clin Neurosci. doi:10.1007/s00406-015-0597-x

4. Hirjak D, Thomann PA, Kubera KM, Stieltjes B, Wolf RC (2015) Cerebellar contributions to neurological soft signs in healthy young adults. Eur Arch Psychiatry Clin Neurosci. doi:10.1007/ s00406-015-0582-4

5. Dowiasch S, Backasch B, Einhäuser W, Leube D, Kircher T, Bremmer F (2014) Eye movements of patients with schizophrenia in a natural environment. Eur Arch Psychiatry Clin Neurosci. doi:10.1007/s00406-014-0567-8

6. Molina V, Bachiller A, Suazo V, Lubeiro A, Poza J, Hornero R (2014) Noise power associated with decreased task-induced variability of brain electrical activity in schizophrenia. Eur Arch Psychiatry Clin Neurosci. doi:10.1007/s00406-014-0569-6

7. Tonna M, Ottoni R, Paglia F, Ossola P, De Panfilis C, Marchesi C (2015) Obsessive-compulsive symptom severity in schizophrenia: a Janus Bifrons effect on functioning. Eur Arch Psychiatry Clin Neurosci. doi:10.1007/s00406-015-0608-y

8. Zhang T, Yi Z, Li H, Cui H, Tang Y, Lu X, Xu L, Qian Z, Zhu Y, Jiang L, Chow A, Li C, Jiang K, Xiao Z, Wang J (2015) Faux pas recognition performance in a help-seeking population at clinical high risk of psychosis. Eur Arch Psychiatry Clin Neurosci. doi:10.1007/s00406-015-0615-Z

9. Xu Z, Müller M, Heekeren K, Theodoridou A, Dvorsky D, Metzler S, Brabban A, Corrigan PW, Walitza S, Rössler W, Rüsch N (2015) Self-labelling and stigma as predictors of attitudes towards help-seeking among people at risk of psychosis: 1-year follow-up. Eur Arch Psychiatry Clin Neurosci. doi:10.1007/ s00406-015-0576-2

10. Hashimoto K (2015) Serine enantiomers as diagnostic biomarkers for schizophrenia and bipolar disorder. Eur Arch Psychiatry Clin Neurosci. doi:10.1007/s00406-015-0602-4 